\title{
A 10-gene signature as a predictor of biochemical recurrence after radical prostatectomy in patients with prostate cancer and a Gleason score $\geq 7$
}

\author{
XIANGKUN WU ${ }^{1,2^{*}}$, DAOJUN LV ${ }^{1,2^{*}}$, MING LEI $^{1,2}$, CHAO CAI $^{1,2}$, ZHIJIAN ZHAO $^{1,2}$, \\ MD EFTEKHAR $^{3}$, DI GU ${ }^{1,2}$ and YONGDA LIU ${ }^{1,2}$ \\ ${ }^{1}$ Department of Urology, Minimally Invasive Surgery Center, The First Affiliated Hospital of Guangzhou Medical University; \\ ${ }^{2}$ Guangdong Key Laboratory of Urology, Guangzhou Institute of Urology, Guangzhou, Guangdong 510230; \\ ${ }^{3}$ Department of Family Medicine, CanAm International Medical Center, Shenzhen, Guangdong 518067, P.R. China
}

Received November 30, 2019; Accepted May 28, 2020

DOI: $10.3892 / \mathrm{ol} .2020 .11830$

\begin{abstract}
The time and speed of biochemical recurrence (BCR) of prostate cancer (PCa) after radical prostatectomy (RP) is highly variable. Stratification methods based on TNM staging and Gleason score (GS) do not allow the identification of patients at risk of BCR following RP. Therefore, the aim of the present study was to identify molecular signatures that can predict $\mathrm{BCR}$ risk effectively and facilitate treatment-related decisions for patients with PCa. RNA sequencing data and corresponding clinical data were downloaded from The Cancer Genome Atlas (TCGA) and Oncomine databases. Bioinformatics analysis was performed to identify differentially expressed genes in patients with $\mathrm{GS}=6$ and $\mathrm{GS} \geq 7$. Cox regression models were used to determine the $\mathrm{PCa}$ signature (PCasig) and a clinical nomogram for the prediction of BCR. The performance of nomograms was assessed using time-dependent receiver operating characteristic curves and the concordance index (C-index). A PCasig comprising 10 genes, including SEMG2, KCNJ16, TFAP2B, SYCE1, KCNU1, AFP, GUCY1B2, GRIA4, NXPH1 and SOX11, was significantly associated with BCR, which was identified in TCGA cohort [hazard ratio (HR), 5.18; 95\% CI, 3.241-8.272; C-index, 0.777 ] and validated in the Oncomine cohort (HR, 2.78; 95\% CI, 1.39-5.54; C-index, 0.66). The expression levels of SEMG2, KCNJ16 and TFAP2B were downregulated in patients with
\end{abstract}

Correspondence to: Professor Yongda Liu or Dr Di Gu, Department of Urology, Minimally Invasive Surgery Center, The First Affiliated Hospital of Guangzhou Medical University, 1 Kangda Road, Guangzhou, Guangdong 510230, P.R. China E-mail: 13719007083@163.com

E-mail: di.gu@nhs.net

*Contributed equally

Key words: prostate cancer, biochemical recurrence, bioinformatics analysis, 10-gene signature
GS $\geq 7$. The expression levels of SYCE1, KCNU1, AFP, GUCY1B2, GRIA4, NXPH1 and SOX11 were upregulated in patients with $\mathrm{GS} \geq 7$. The clinical nomogram was constructed based on the GS and pathologic T stage (HR, 4.15; 95\% CI, 1.39-5.54; C-index, 0.713). The addition of the PCasig to the clinical nomogram significantly improved prognostic value (HR, 7.25; 95\% CI, 4.54-11.56; C-index, 0.782) with an net reclassification improvement of $75.3 \%$ (95\% CI, 46.8-104.6\%). Furthermore, the endogenous expression of each gene in the PCasig was measured in five PCa cell lines and in normal prostate cells, and these genes exhibited different expression levels relative to one another. In conclusion, an PCasig was identified by mining TCGA and successfully validated in an Oncomine cohort. This PCasig was an independent prognostic factor with a greater prognostic value for all patients regardless of GS than traditional clinical variables, which can improve the performance of clinical nomograms in predicting BCR of patients with $\mathrm{GS} \geq 7$.

\section{Introduction}

Prostate cancer (PCa) is the most common malignant tumor and the second leading cause of cancer-associated mortality among men in the Western world (1). In 2015, 60,300 new cases of PCa were diagnosed in China, leading to 26,000 mortalities (2). In patients treated with radical prostatectomy (RP) or radiation therapy for localized $\mathrm{PCa}$, biochemical recurrence (BCR) is defined as two consecutive prostate-specific antigen (PSA) values $\geq 0.2 \mathrm{ng} / \mathrm{ml}$ after $\mathrm{RP}$, or a PSA level $\geq 2.0 \mathrm{ng} / \mathrm{ml}$ above the nadir after external beam radiation therapy or brachytherapy (3). The rate of BCR following RP has been estimated to be $20-40 \%$ (4). In the absence of secondary treatment, patients with BCR have an approximate median period of 5-8 years prior to clinical progression; among these, 32-45\% will succumb to PCa within 15 years (5).

In order to facilitate BCR assessment, evaluate prognosis and individualize patient follow-up, it is critical to identify patients with a high-risk of BCR following RP. However, the current risk stratification methods, which are mainly based on clinicopathological parameters, only partially address the 
observed variation in clinical outcomes and are not accurate enough to predict high-risk BCR (6). With the development of next-generation sequencing, several genomic biomarkers have been identified and used as prognostic factors and predictive signatures $(7,8)$.

The Gleason system was developed by Donald Gleason between 1966-1974 and was used to evaluate the degree of malignance of $\mathrm{PCa}$ (9). In previous years, multiple studies have described that PCa with a Gleason score (GS) of 6 is unable to metastasize or cause cancer-associated mortality, in addition to having a low risk rate for BCR after RP (10-12). Although patients with $G S \geq 7$ are at a much higher risk for disease progression compared with patients with $\mathrm{GS}=6$, even patients with GS $\geq 8$ can experience favorable oncological outcomes, such as BCR risk and survival time, which highlights the heterogeneity of PCa $(13,14)$. Furthermore, patients with GS $3+4 \mathrm{PCa}$ have similar outcomes compared with patients with $\mathrm{GS}=6$ (15). Therefore, it was hypothesized that the genomic features of GS $=6 \mathrm{PCa}$, which may also be shared with GS $\geq 7$ $\mathrm{PCa}$, could be harnessed to further stratify PCa into conventional, intermediate and high-risk PCa.

The aim of the present study was to identify differentially expressed genes (DEGs) between patients with PCa and GS=6 and GS $\geq 7$, using The Cancer Genome Atlas (TCGA) data. These DEGs were used to construct and validate an $\mathrm{PCa}$ signature (PCasig) that could identify patients with a high-risk of BCR using Cox regression analysis. In addition, the present study aimed to analyze the biological signaling pathway associated with the PCasig using Gene Ontology (GO) and Kyoto Encyclopedia of Genes and Genomes (KEGG) pathway enrichment analysis. Lastly, the endogenous expression of the genes identified in the PCasig was determined in PCa and normal prostate cell lines.

\section{Materials and methods}

Data preparation and processing. A flowchart of the analysis conducted in the present study is shown in Fig. 1. High-throughput RNA sequencing (RNA-seq) data, with RNA-seq data (HTSeq-Counts) and corresponding clinicopathological parameters of patients with PCa after RP were obtained from TCGA (https://tcga-data.nci.nih.gov/tcga/), and served as the discovery cohort (TCGA-PRAD). Taylor Prostate 3, another gene expression array of human PCa datasets, was obtained from Oncomine (https://www.oncomine. org/resource/login.html) and served as the validation cohort. The inclusion criteria were as follows: i) Pathologically confirmed diagnosis of prostate adenocarcinoma; ii) complete record of clinicopathological parameters, including follow-up time, age, GS, pathological $\mathrm{T}$ and $\mathrm{N}$ stage, clinical $\mathrm{M}$ stage, situation of residual tumor, and time to BCR; and iii) RNA-seq data. The exclusion criteria were as follows: i) Pathologic result was not prostate adenocarcinoma; ii) missing clinicopathological parameters, including follow-up time, age, GS, pathological $\mathrm{T}$ and $\mathrm{N}$ stage, clinical $M$ stage (16), situation of residual tumor, and time to BCR; and iii) no RNA-seq data.

A total of 414 patients with both RNA-seq data and corresponding clinicopathological information were included in the discovery cohort and 377 of them had a GS $\geq 7$. The median age of patients in the discovery cohort was 61 years (age range, 41-78 years). A total of 138 patients with RNA-seq data and corresponding clinicopathological parameters were included in the validation cohort, and 97 of them had a GS $\geq 7$. The median age of patients in the validation cohort was 58 years (age range, $37-73$ years).

Development and validation of the PCasig. The DEGs in $\mathrm{GS}=6$ and $\mathrm{GS} \geq 7$ PCa were analyzed in the discovery cohort using the edgeR package (version 3.30.0; http://www.bioconductor.org/packages/release/bioc/html/edgeR.html) in $\mathrm{R}$ software (version 3.5.2; https://www.r-project.org), with Ilog2 [fold change (FC)] $>1.0$ and a false discovery rate of 0.05 used as the threshold values. The significant DEGs were considered to be candidate genes for further analysis.

The RNA-seq data were normalized using the transcripts per million (TPM) method and a $\log 2$-based transformation ( $\log _{2}$ TPM) for further survival analysis. Univariate Cox proportional hazards $(\mathrm{PH})$ regression analysis was used to analyze the association between the expression levels of the DEGs and time to BCR in patients with $\mathrm{GS} \geq 7$. $\mathrm{P}<0.05$ was considered to indicate a statistically significant difference. The least absolute shrinkage and selection operator (LASSO) method was utilized to screen significant DEGs and to avoid overfitting the model. The LASSO method can effectively solve the computational difficulties of estimation in high-dimensional and low-sample size environments (17).

Based on the results of the LASSO analysis, multivariate Cox analysis was performed to identify a PCasig and calculate the risk score of each patient, using weighted estimators corresponding to each gene expression level. All patients were divided into low and high score groups according to the median risk score. Kaplan-Meier curves and a log-rank test were used to assess the prognostic effect of the prognosis risk score. The PCasig was applied to the validation cohort to determine whether it could discriminate high-risk BCR patients. PCasig performance was assessed by time-dependent receiver operating characteristic (ROC) curve analysis within 3 and 5 years, and the concordance index (C-index).

Inthepresentstudy, the 'survival' package was installedforthe R software version 3.5.2 (R Core Team; https://www.R-project. org/). The 'survival' package (v3.1-12; https://cran.r-project. org/web/packages/survival/index.html) was used to perform Cox regression analysis. In addition, the following packages were used: i) 'glmnet' for LASSO Cox analysis (version 4.0; https://cran.r-project.org/web/packages/glmnet/index.html); ii) 'survminer' for survival curves (v0.4.7; https://cran. r-project.org/web/packages/survminer/index.html); iii) 'survival ROC' to obtain the area under the curve (AUC) (v1.0.3; https://cran.r-project.org/web/packages/survivalROC/index.html); andi) 'rms' tocalculate theC-index (v5.1-4; https://cran.r-project. org/web/packages/rms/).

Association and comparison of the PCasig with the clinicopathological parameters. Univariate and multivariate analysis using Cox regression models was conducted in the discovery cohort to test the prognostic effect of each parameter and their dependencies. The present study compared the prognostic performance of the PCasig against the clinical nomogram constructed using clinicopathological parameters with respect 


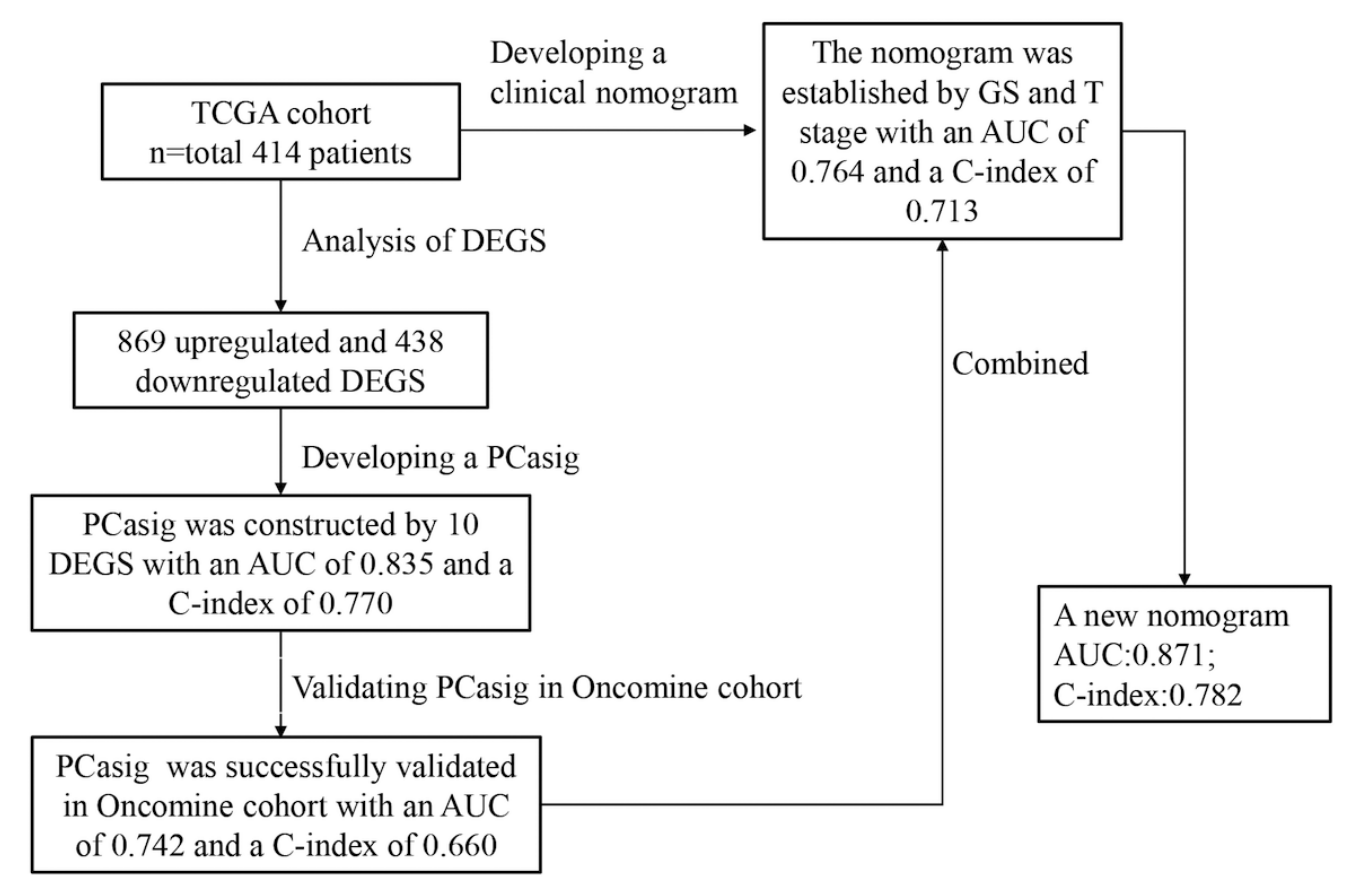

Figure 1. Flow diagram for the present systematic analysis and validation. DEGs, differentially expressed Gs; PCasig, PCa signature; GS, Gleason score; $\mathrm{T}$, pathological $\mathrm{T}$.

to time to BCR. $\mathrm{P}<0.05$ was considered to indicate a statistically significant difference.

Incremental predictive value of the PCasig for the clinical nomogram. In order to evaluate whether the PCasig could improve the predictive performance, a new nomogram was constructed, by adding the PCasig into the clinical nomogram, as a prognostic factor. Therefore, the incremental value of the PCasig as an additional candidate predictor was evaluated, the AUC and the calibration curve were derived, and the net reclassification improvement (NRI) was calculated using 'nricens' package (version 1.6; https://cran.r-project. org/web/packages/nricens/) (18).

Prognostic effect of the PCasig in different GS subgroups. The present study also evaluated whether the PCasig was a significant predictor of BCR compared with other clinicopathological parameters in different GS subgroups. Cox regression and Kaplan-Meier curve analyses were performed in the GS=7 and $\mathrm{GS} \geq 8$ subgroups.

Functional enrichment analysis. GO and KEGG pathway enrichment analysis of PCasig were performed using the 'cluster profiler' package (version 3.16.0; https://bioconductor. org/packages/release/bioc/html/clusterProfiler.html) to examine the GO terms and pathways. $\mathrm{P}<0.05$ was considered to indicate a statistically significant enrichment.

Cell lines and culture. Human PCa cell lines (PC-3, DU145, 22RV1, C4-2 and LNCaP) were purchased from The Cell Bank of Type Culture Collection of the Chinese Academy of Sciences. All the PCa cell lines were cultured in RPMI-1640 medium supplemented with $10 \%$ fetal bovine serum (cat. no. 10099; Gibco, Thermo Fisher Scientific, Inc.). Normal prostatic epithelial cells RWPE-1 were purchased from the American Type Culture Collection and cultured in Keratinocyte Serum Free Medium (cat. no. 10744-019) supplemented with $5 \mathrm{ng} / \mathrm{ml}$ epidermal growth factor (cat. no. 10450-013) and 1\% penicillin/streptomycin (cat. no. 15140-122; all purchased from Gibco; Thermo Fisher Scientific, Inc.). All cells were incubated at $37^{\circ} \mathrm{C}$ in $5 \% \mathrm{CO}_{2}$.

RNA extraction and RT-quantitative PCR assays. Total RNA was extracted from cells with RNAiso Plus reagent (cat. no. 9109; Takara Bio, Inc.) and cDNA was reverse-transcribed using the PrimeScript RT reagent kit (cat. no. RR047A; Takara Bio, Inc.) according to the manufacturer's instructions. The temperature protocol was as follows: $37^{\circ} \mathrm{C}$ for $15 \mathrm{~min}$ and $85^{\circ} \mathrm{C}$ for $5 \mathrm{sec}$ for one cycle. Quantitative PCR analysis was performed using the One Step TB Green ${ }^{\circledR}$ PrimeScript $^{\mathrm{TM}}$ PLUS RT-PCR kit (Perfect Real Time) (cat. no. RR096A; Takara Bio, Inc.), with a 7500 Fast Real-Time RCR system (Applied Biosystems; Thermo Fisher Scientific, Inc.). The thermocycling conditions were as follows: $95^{\circ} \mathrm{C}$ for $5 \mathrm{sec}, 55^{\circ} \mathrm{C}$ for $30 \mathrm{sec}, 72^{\circ} \mathrm{C}$ for $30 \mathrm{sec}$ and repeats for 40 cycles. Each measurement was performed in triplicate and the results were normalized to the GAPDH internal control. The $2^{-\Delta \Delta \mathrm{Cq}}$ method was used to calculate the relative expression levels of target genes (19). All primer sequences are presented in Table SI.

Statistical analysis. Statistical analyses were conducted using SPSS version 19.0 software (IBM Corp.). All experiments were performed in triplicate and numerical data are presented as the mean \pm standard deviation. Differences among PCa and normal prostate cells were analyzed by one-way ANOVA, and the Dunnett test was used as the post hoc test. Kaplan-Meier curves and the log-rank tests were used to assess associations between PCaSig expression and BCR. Cox proportional 


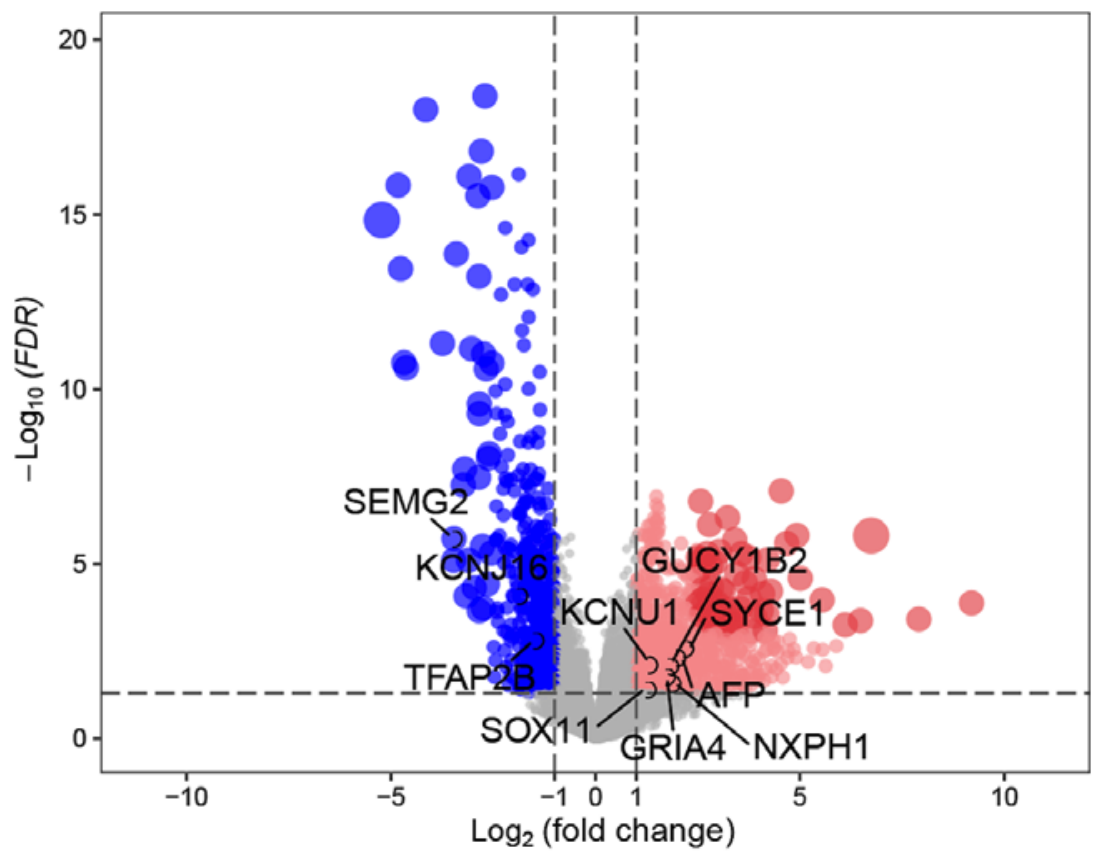

Figure 2. Volcano plots of differentially expressed genes in tissues from patients with GS=6 and tissues from patients with GS $\geq 7$. The $x$-axis represents the $\log 2$-scaled fold change, whereas the $y$-axis indicates the log-scaled P-value. Each symbol corresponds to a different gene, with red dots representing upregulated genes and blue dots representing downregulated genes according to the following thresholds: Adjusted P $<0.05$; and $\log 2$ (fold change) $=1$. GS, Gleason score; FDR, false discovery rate; KCNU1, potassium calcium-activated channel subfamily U member 1; SEMG2, semenogelin 2; SOX11, SRY-box transcription factor 11; KCNJ16, potassium inwardly rectifying channel subfamily J member 16; AFP, $\alpha$-fetoprotein; GUCY1B2, guanylate cyclase 1 soluble subunit $\beta 2$ (pseudogene); TFAP2B, transcription factor AP-2 $\beta$; GRIA4, glutamate ionotropic receptor AMPA type subunit 4; SYCE1, synaptonemal complex central element protein 1; NXPH1, neurexophilin 1.

A 160 149

118

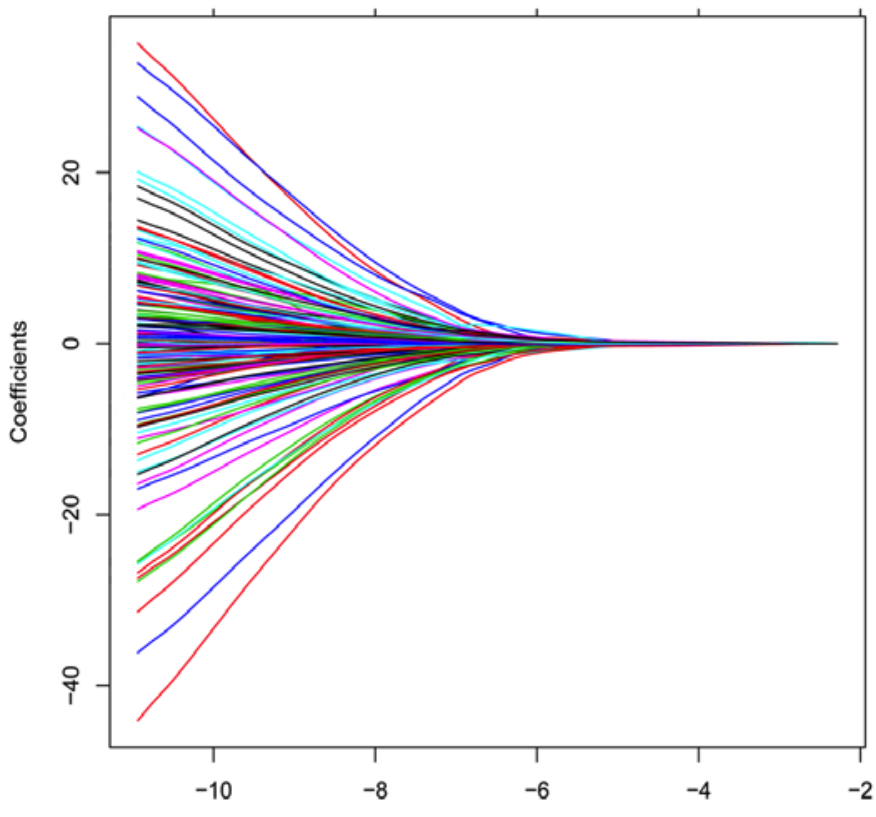

Log Lambda
○B $\begin{array}{llllllllllllll}168 & 160 & 154 & 152 & 147 & 145 & 138 & 107 & 85 & 56 & 39 & 38 & 11 & 1\end{array}$

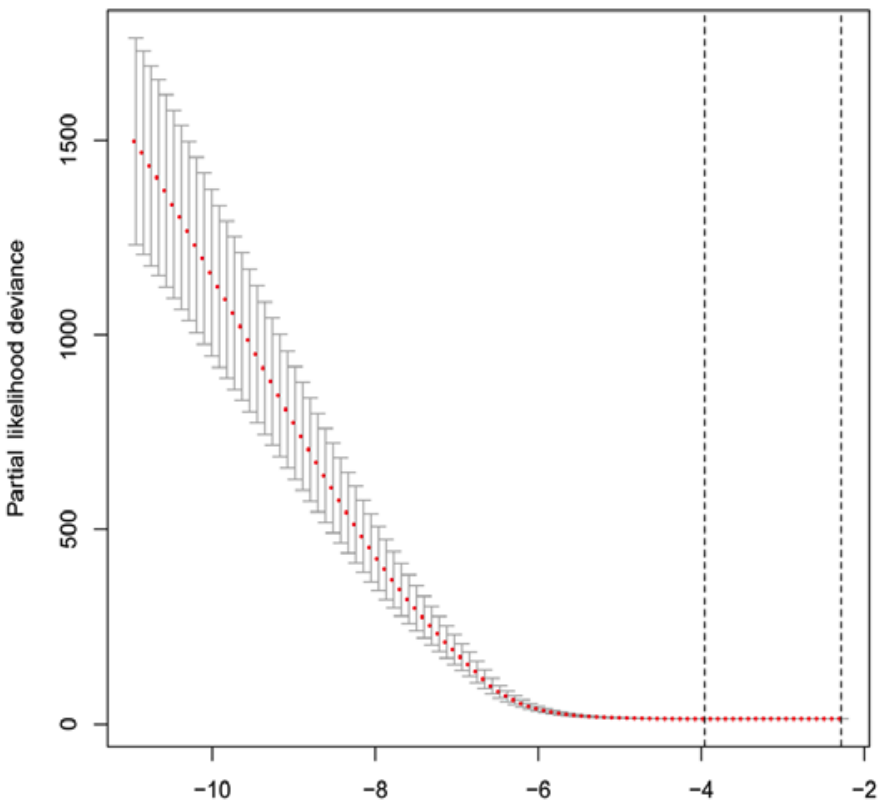

Log Lambda

Figure 3. LASSO Cox analysis via 10-fold cross-validation with minimum criteria. (A) Tuning parameter selection via 10 -fold cross-validation with minimum criteria in the LASSO model. (B) LASSO coefficient profiles of 171 prognostic genes. LASSO, least absolute shrinkage and selection operator.

hazards analysis was performed to assess the relative impacts of different groups (high vs. low level) on BCR. The concordance index (C-index) was used to evaluate the discriminatory powers of the signature. And calibration plot was used as an internal validation of the nomogram. $\mathrm{P}<0.05$ was considered to indicate a statistically significant difference. 
A

Hazard ratio

\begin{tabular}{|c|c|c|c|c|c|}
\hline SEMG2 & $(N=377)$ & $\begin{array}{c}0.85 \\
(0.77-0.94)\end{array}$ & $\longmapsto$ & & 0.001 ** \\
\hline KCNJ16 & $(N=377)$ & $\begin{array}{c}0.78 \\
(0.66-0.92)\end{array}$ & $\longmapsto$ & & $0.004 * *$ \\
\hline TFAP2B & $(N=377)$ & $\begin{array}{c}0.72 \\
(0.59-0.89)\end{array}$ & & & $0.002^{* *}$ \\
\hline SYCE1 & $(N=377)$ & $\begin{array}{c}0.87 \\
(0.78-0.97)\end{array}$ & $\longmapsto$ & & $0.016^{*}$ \\
\hline AFP & $(N=377)$ & $\begin{array}{c}1.20 \\
(1.07-1.36)\end{array}$ & & $\longmapsto$ & $0.002 * *$ \\
\hline KCNU1 & $(N=377)$ & $\begin{array}{c}0.79 \\
(0.68-0.93)\end{array}$ & - & & 0.004 ** \\
\hline GUCY1B2 & $(N=377)$ & $\begin{array}{c}1.19 \\
(1.07-1.31)\end{array}$ & & $\longmapsto$ & $0.001^{* *}$ \\
\hline GRIA4 & $(N=377)$ & $\begin{array}{c}1.22 \\
(1.08-1.36)\end{array}$ & & & $<0.001$ ** \\
\hline NXPH1 & $(N=377)$ & $\begin{array}{c}1.24 \\
(1.10-1.40)\end{array}$ & & & $<0.001^{* *}$ \\
\hline sox11 & $(N=377)$ & $\begin{array}{c}1.34 \\
(1.17-1.55)\end{array}$ & & & $-1<0.001$ ** \\
\hline \multicolumn{6}{|c|}{ HEvents: 71; Global P-value (log-rark): 2.6447e-13 } \\
\hline IIC: 717.57; & cordance & index: $0.78_{0}$ & 0.8 & 1.2 & 1.6 \\
\hline
\end{tabular}

B

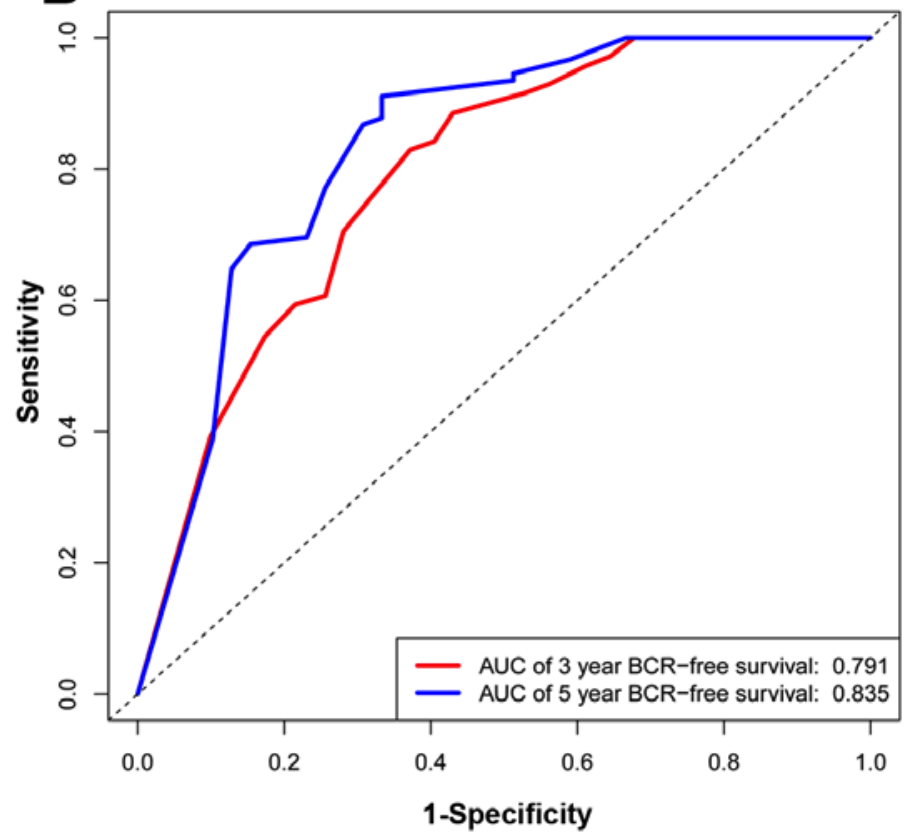

C

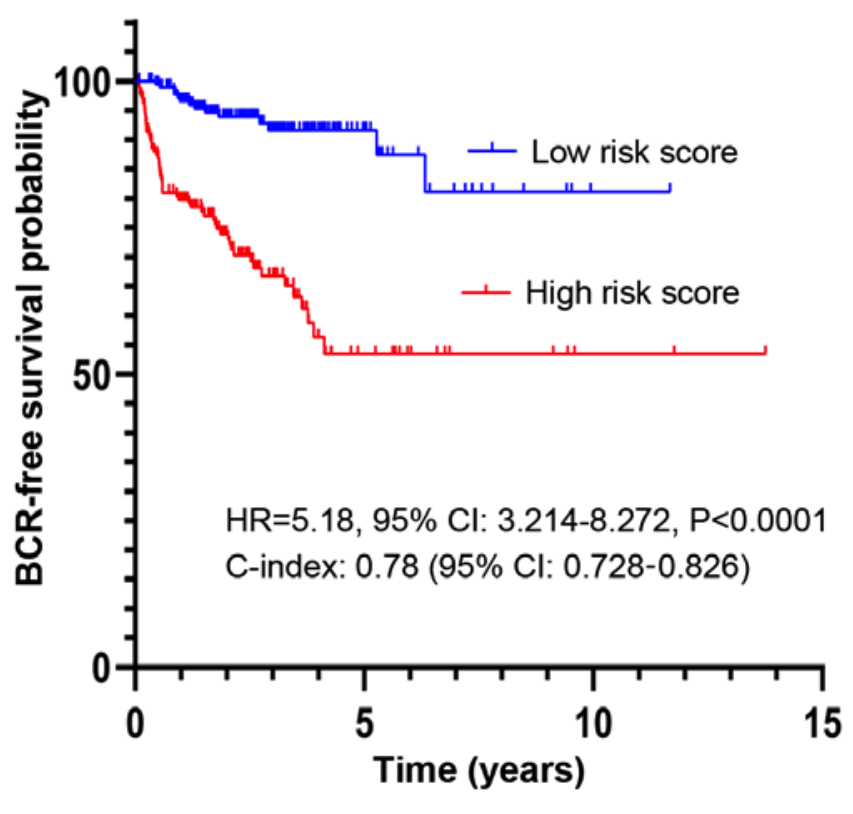

Figure 4. Risk score performance in the training datasets. (A) Forest plot representing 10 genes identified as independent factors for BCR prediction using multivariate Cox analysis. (B) ROC curve based on the PCasig in the discovery cohort. (C) Kaplan-Meier curves of the PCasig in the discovery cohort to predict BCR-free survival. BCR, biochemical recurrence; AUC, area under the curve; ROC, receiver operating characteristic; HR, hazard ratio; C-index, concordance index.

\section{Results}

Differential gene expression profiles. A total of 1,307 DEGs between the GS=6 and GS $\geq 7$ groups were identified in the TCGA discovery cohort, according to the cut-off points of
$\mathrm{P}<0.05$ and $|\log 2(\mathrm{FC})|>1.0$. Among these, 869 were upregulated and 438 were downregulated (Fig. 2).

Development and validation of the PCasig. The clinicopathological characteristics in both the discovery and validation 
Table I. Multivariate HR analysis for clinicopathological variables and risk score in The Cancer Genome Atlas cohort.

\begin{tabular}{|c|c|c|c|c|}
\hline \multirow[b]{2}{*}{ Variable and intercept } & \multicolumn{2}{|c|}{ Multivariate Cox 1} & \multicolumn{2}{|c|}{ Multivariate Cox 2} \\
\hline & $\operatorname{HR}(95 \% \mathrm{CI})$ & P-value & $\mathrm{HR}(95 \% \mathrm{CI})$ & P-value \\
\hline \multicolumn{5}{|l|}{ GS } \\
\hline $3+4$ & 1.00 & & 1.00 & \\
\hline $4+3$ & $2.56(0.87-7.51)$ & 0.046 & $2.21(0.77-6.36)$ & 0.09200 \\
\hline$\geq 8$ & $3.97(1.46-10.73)$ & 0.0020 & $3.80(1.45-9.93)$ & 0.00650 \\
\hline \multicolumn{5}{|l|}{ PT } \\
\hline $\mathrm{T} 2$ & 1.00 & & 1.00 & \\
\hline $\mathrm{T} 3 \mathrm{a}$ & $1.99(0.77-5.08)$ & 0.020 & $2.94(1.18-7.25)$ & 0.02000 \\
\hline $\mathrm{T} 3 \mathrm{~b}+\mathrm{T} 4$ & $2.84(1.09-7.37)$ & 0.0030 & $3.44(1.40-8.48)$ & 0.00700 \\
\hline \multicolumn{5}{|l|}{$\mathrm{PN}$} \\
\hline N0 & 1.00 & & 1.00 & \\
\hline N1 & $135\left(0-4.19 \times 10^{55}\right)$ & 0.91 & $120.00\left(0.00-3.19 \times 10^{45}\right)$ & 0.92000 \\
\hline NX & $0.47(0.11-1.94)$ & 0.29 & $0.37(0.11-1.04)$ & 0.39000 \\
\hline \multicolumn{5}{|l|}{ Risk score } \\
\hline Low & NA & & 1.00 & \\
\hline High & NA & & $3.34(1.87-6.00)$ & $<0.00010$ \\
\hline
\end{tabular}

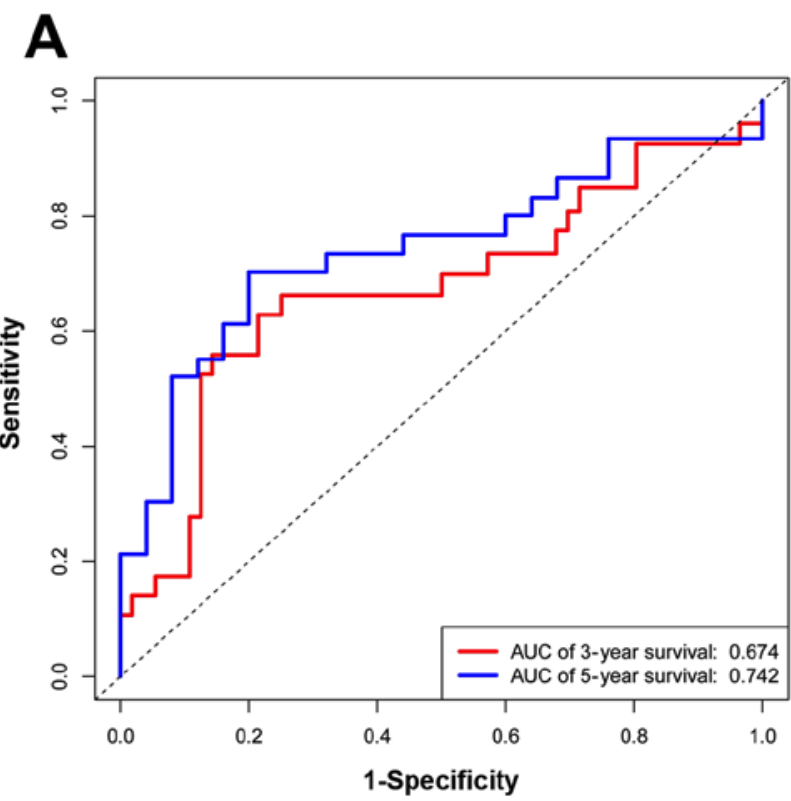

B

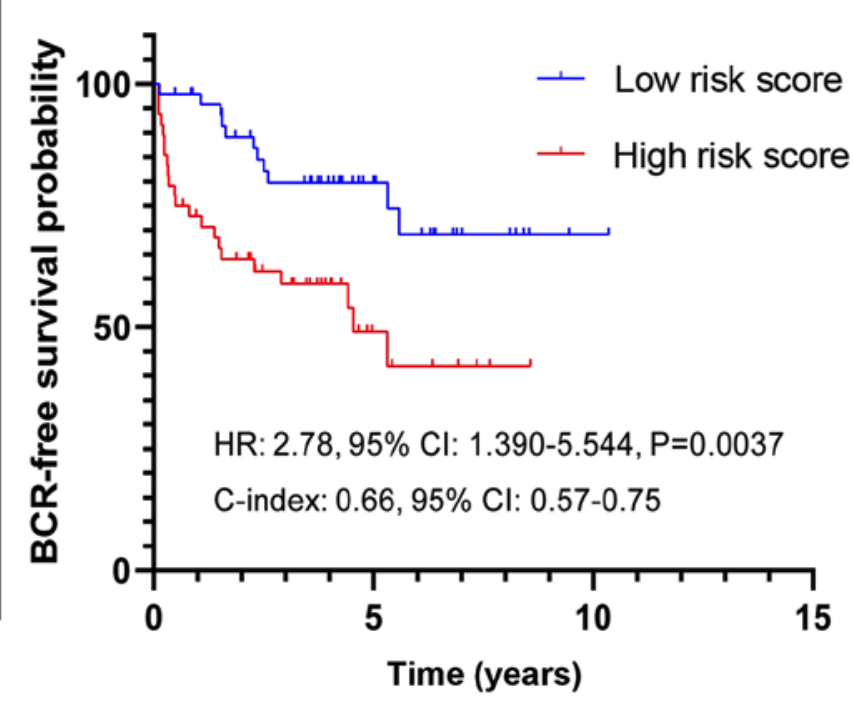

Figure 5. Validation of the PCasig in Oncomine cohort. (A) Receiver operating characteristic curve based on the PCasig. (B) Kaplan-Meier curve of the PCasig to predict BCR-free survival. PCasig, PCa signature; BCR, biochemical recurrence; HR, hazard ration; CI, confidence interval; C-index, concordance index.

cohorts are presented in Table SII. The mean follow-up time was 31.4 months (range, 1.0-167.5 months) and 43.0 months (range 1.4-126.0 months), respectively. Among the 1,307 DEGs, 171 prognostic genes were identified using univariate Cox regression analysis in TCGA cohort, while 39 prognostic genes were identified by LASSO Cox using 10-fold cross-validation with minimum criteria (Fig. 3).
A total of 10 prognostic genes, including potassium calcium-activated channel subfamily U member 1 (KCNU1), semenogelin 2 (SEMG2), SRY-box transcription factor 11 (SOX11), potassium inwardly rectifying channel subfamily $\mathbf{J}$ member 16 (KCNJ16), $\alpha$-fetoprotein (AFP), guanylate cyclase 1 soluble subunit $\beta 2$ (GUCY1B2), transcription factor AP-2 $\beta$ (TFAP2B), glutamate ionotropic receptor AMPA type subunit 


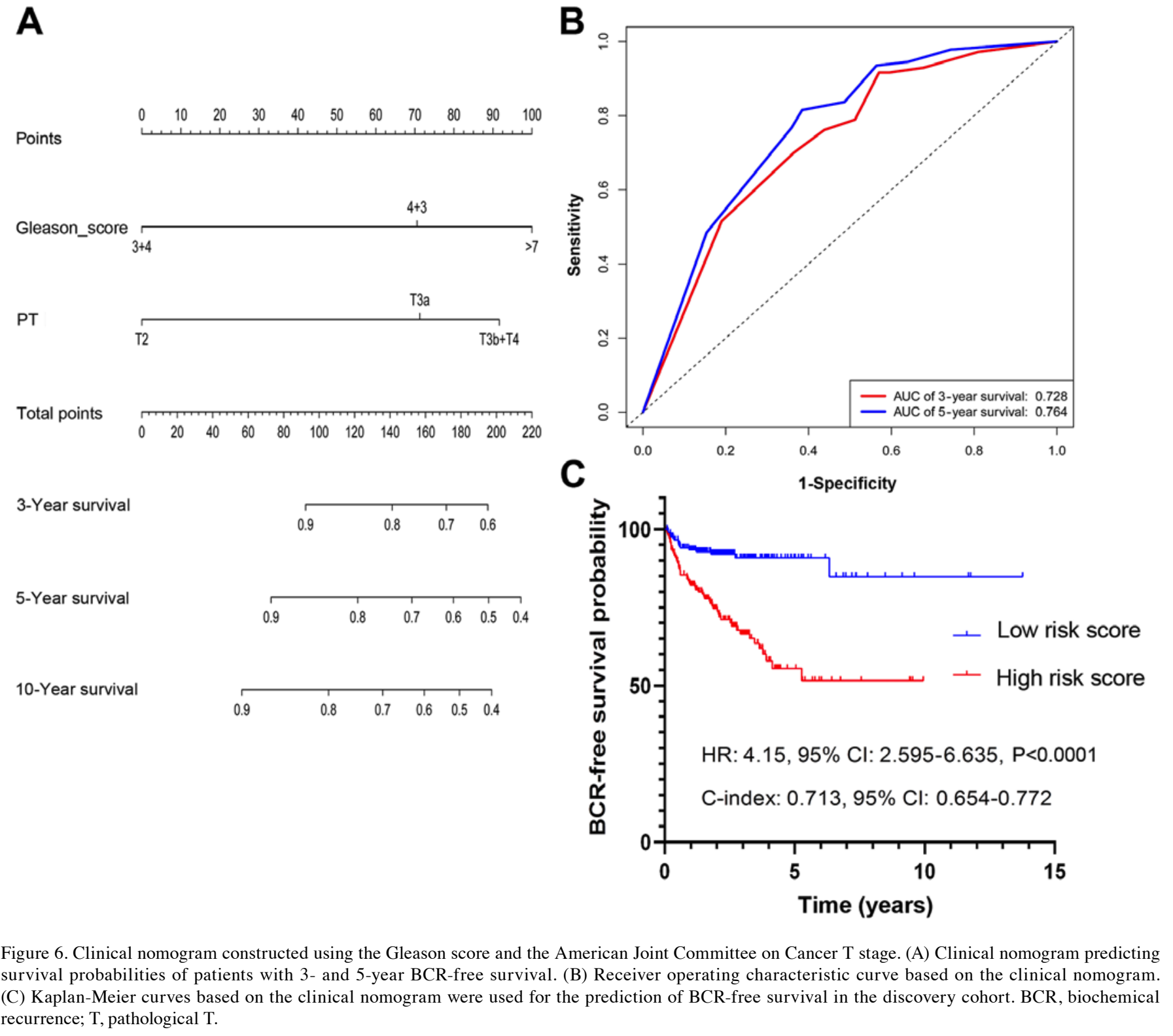

4 (GRIA4), synaptonemal complex central element protein 1 (SYCE1) and neurexophilin 1 (NXPH1), were identified by multivariate Cox regression analysis (Fig. 4A).

SEMG2, KCNJ16, TFAP2B, SYCE1 and KCNU1 were associated with a hazard ratio (HR) $<1$. By contrast, AFP, GUCY1B2, GRIA4, NXPH1 and SOX11 had a HR $>1$. The differential expression of the 10 prognostic genes between patients with $\mathrm{GS}=6$ and patients with $\mathrm{GS} \geq 7$ is presented as a heatmap (Fig. S1).

The AUC for the PCasig was 0.791 and 0.835 for 3- and 5 -year BCR-free survival, respectively (Fig. 4B). The PCasig had a C-index of 0.78 (95\% CI, 0.728-0.826).

The risk score for each patient was calculated as follows: $(-0.16 \mathrm{x}$ SEMG2 expression level $)+(-0.25 \mathrm{x}$ KCNJ16 expression level $)+(-0.321 \mathrm{x}$ TFAP2B expression level $)+(-0.14 \mathrm{x}$ SYCE1 expression level $)+(-0.23 \mathrm{x}$ KCNU1 expression level $)+(0.19 \mathrm{x}$ AFP expression level $)+(0.17 \mathrm{x}$ GUCY1B2 expression level $)+$ (0.20x GRIA4 expression level) $+(0.22 \mathrm{x}$ NXPH1 expression level $)+(0.30 x$ SOX11 expression level). A total of 377 patients with GS $\geq 7$ were divided into a high and a low-score group based on the median of the score distribution. This stratification of the risk score was associated with a hazard ratio (HR) of 5.18 (high vs. low; 95\% CI, 3.241-8.272; P<0.0001; Fig. 4C).

In the validation cohort, the PCasig identified patients with a high-risk of BCR with an AUC of 0.761 for 5-year BCR-free survival time (Fig. 5A) and a C-index of 0.66. The BCR risk of GS $\geq 7$ patients in the high-score group was significantly higher compared with that in the low-score group (HR, 2.78; 95\% CI, 1.39-5.54; P=0.0037; Fig. 5B).

Association and comparison of the PCasig with clinicopathological parameters. PCasig and clinicopathological parameters were analyzed using univariate and multivariate Cox regression analysis (Tables I and SIII). Regarding the $\mathrm{PH}$ Assumption, the P-value of seven factors, including age, GS, $\mathrm{T}$, stage, $\mathrm{N}$ stage, Clinical $\mathrm{M}$, residual tumor and the PCasig, was $>0.05$ and the $\mathrm{PH}$ assumption could not be considered violated. The PCasig, GS and T stage were identified as independent predictors of BCR following RP in patients with $\mathrm{GS} \geq 7$. 
A

Points

PT

Gleason_score

Risk_level

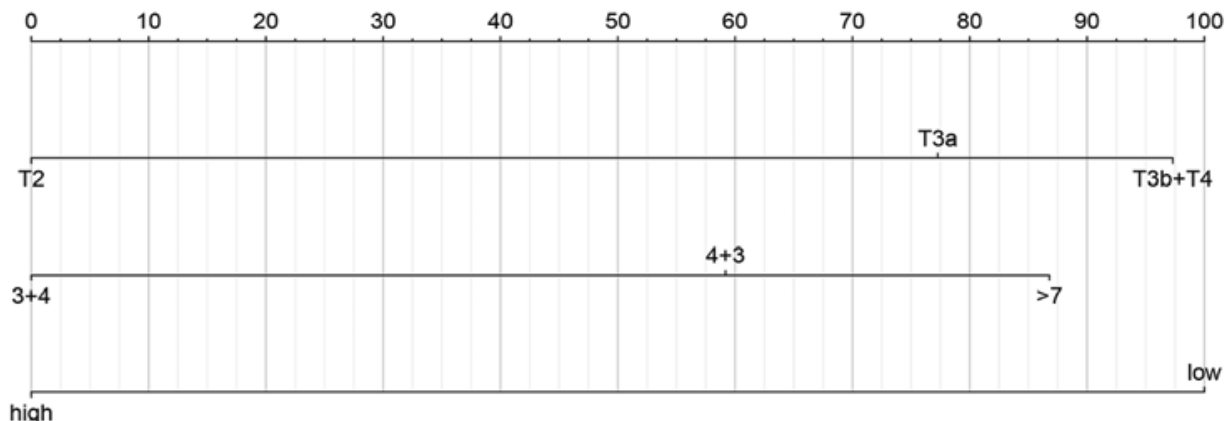

Total points

$$
0
$$

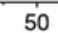

100

150

200

250

300

350

3-Year BCR-free survival probability

$\begin{array}{lllllll}0.95 & 0.9 & 0.8 & 0.7 & 0.6 & 0.5 & 0.4\end{array}$

5-Year BCR-free survival probability

B

\begin{tabular}{llllllll}
\hline 0.95 & 0.9 & 0.8 & 0.7 & 0.6 & 0.5 & 0.4 & 0.3
\end{tabular}
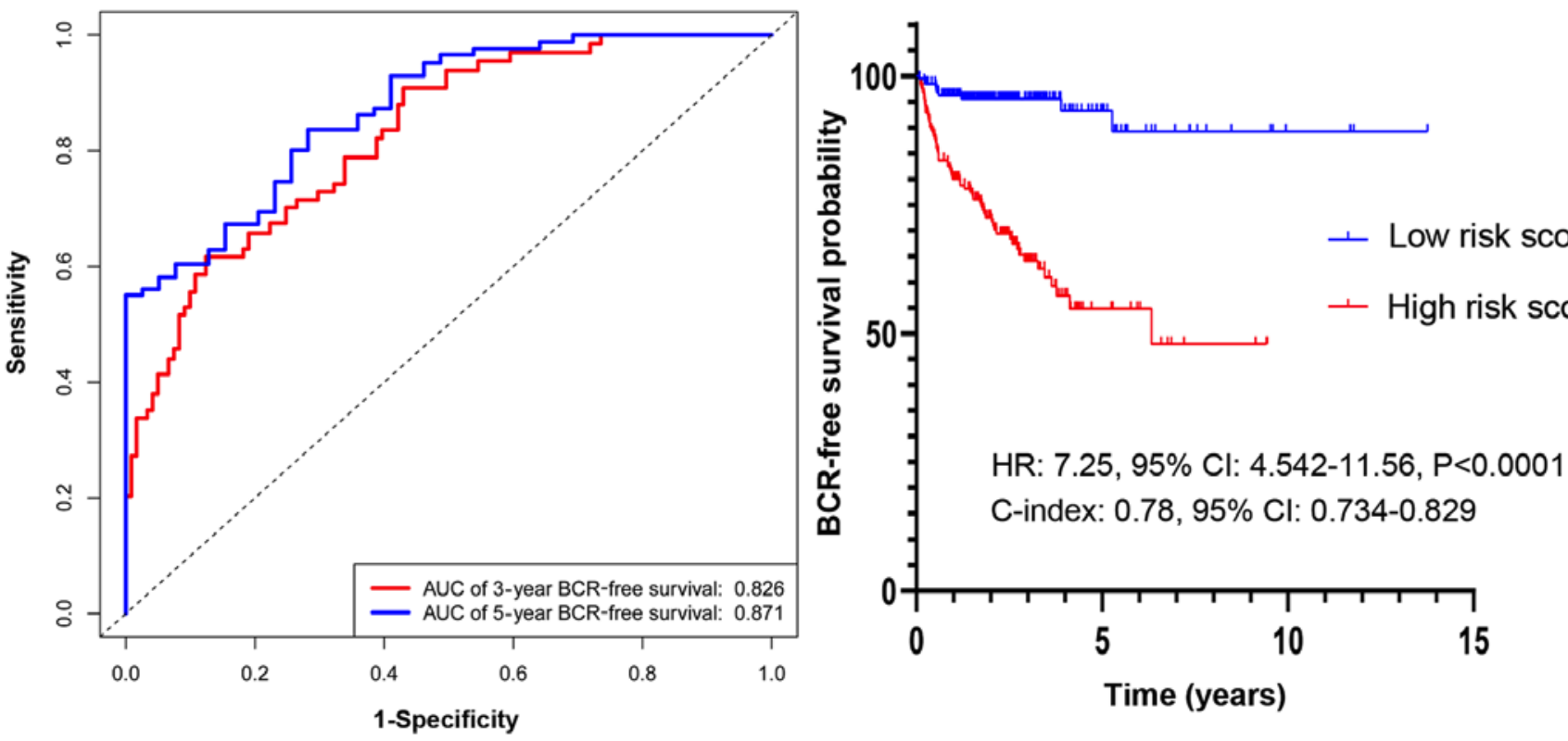

Figure 7. Nomogram constructed using the risk score, Gleason score and the American Joint Committee on Cancer T stage. (A) Nomogram predicting survival probabilities of patients with 3- and 5-year BCR-free survival. (B) Receiver operating characteristic curve based on the new nomogram. (C) Kaplan-Meier curves based on the new nomogram were used to determine the association between the risk score and survival. BCR, biochemical recurrence; AUC, area under the curve; HR, hazard ratio; PT, pathological T; C-index, concordance index.

A clinical nomogram was constructed based on the GS and T stage (Fig. 6A). The AUCs at the 3- and 5-year BCR-free survival time points were 0.726 and 0.764 , respectively (Fig. 6B). The C-index of the nomogram was 0.713 (95\% CI, 0.654-0.772). The BCR of patients with GS $\geq 7$ in the high risk score group was significantly higher, compared with that of patients in the low score group (HR, 4.15; 95\% CI, 2.56-64; P<0.001; Fig. 6C).

Incremental predictive value of the PCasig for the clinical nomogram. A new nomogram was constructed and included the risk score, in addition to GS and T stage (Fig. 7A). With the PCasig, the new nomogram yielded an AUC of 0.826 at the 3-year BCR-free survival time point, and 0.871 at the 5-year time point (Fig. 7B). The C-index was 0.78 (95\% CI, $0.734-0.829$ ). The BCR risk of patients with $\mathrm{GS} \geq 7$ in the high risk score group was significantly higher compared with that of patients in the low score group (HR, 7.25; 95\% CI, 4.54-11.56; $\mathrm{P}<0.0001$; Fig. 7C). The BCR-free survival rate predicted using the new nomogram was positively associated with the actual observed rate (Fig. 8A and B).

The NRI (NRI, 0.75; P<0.0001, event NRI, 0.66; non-event NRI, 0.09) indicated that the performance was significantly improved after the integration of the PCasig into the clinical nomogram. For patients with BCR, the proportion 

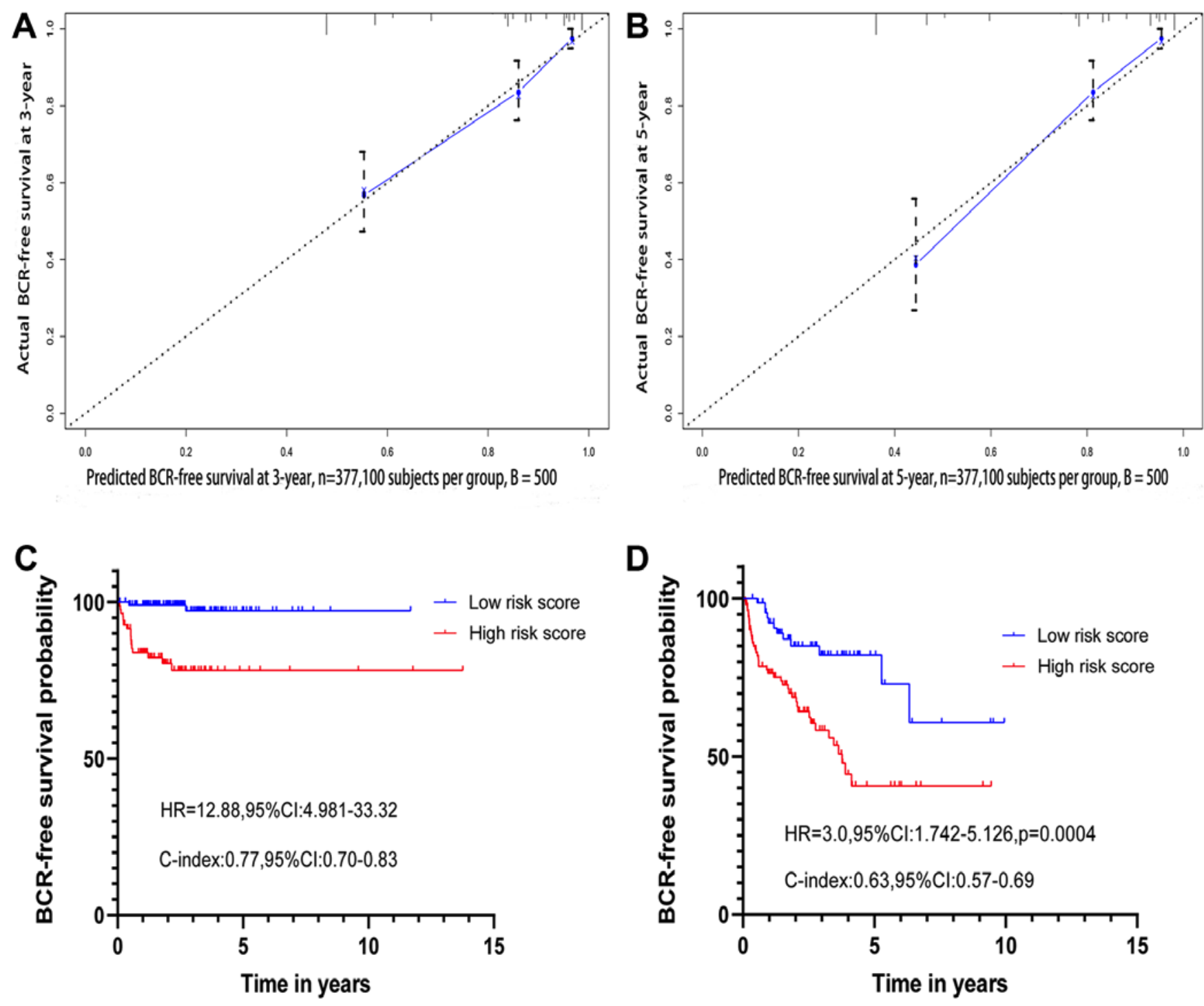

Figure 8. Validation of the new nomogram in predicting overall survival of PCa in the TCGA cohort. Calibration curves of the new nomogram predicting BCR-free survival at (A) 3 and (B) 5 years. (C and D) Prognostic effect of risk score in different GS subgroups. (C) Kaplan-Meier analysis of BCR-free survival in the high-risk and low-risk subgroups within TCGA patient cohort with GS=7. (D) Kaplan-Meier analysis of patients with GS $\geq 8$ in TCGA cohort. BCR, biochemical recurrence; HR, hazard ratio; TCGA, The Cancer Genome Atlas; C-index, concordance index.

of reclassification significantly increased by $0.66(\mathrm{P}<0.001)$, indicating an improvement of $66 \%$ with the incorporation of the PCasig. For BCR-free patients, the proportion of reclassification significantly increased by $0.09(\mathrm{P}<0.001)$, indicating an improvement of 9\% (Fig. S2).

Prognostic effect of the risk score in different GS subgroups. When limiting the univariate and multivariate analyses to patients with GS=7 in the discovery cohort, the PCasig remained the most significant predictor of BCR compared with the other clinicopathological parameters analyzed in the present study (HR, 9.29; 95\% CI, 2.08-41.30; $\mathrm{P}=0.0034$; Table II). Additionally, as indicated in Table III, the PCasig remained the most significant predictor of $\mathrm{BCR}$ in the $\mathrm{GS} \geq 8$ subgroup (HR, 3.01; 95\% CI, 1.58-5.75; $\mathrm{P}=0.0008$ ).

The PCasig was used to divide patients with GS=7 $(n=201)$ in the discovery cohort into low- and high-risk groups with a significant BCR difference (high vs. low; HR, 12.88; 95\% CI, 4.981-33.32; $\mathrm{P}<0.0001$ ) with a C-index of 0.77 (Fig. 8C). The
PCasig was also used to divide patients with GS $\geq 8$ into low and high-risk groups, which had a significantly different BCR risk (high vs. low; HR, 3.0; 95\% CI, 1.742-5.126; $\mathrm{P}=0.0004$ ) with a C-index of 0.63 (Fig. 8D).

Functional enrichment analysis. A total of $17 \mathrm{GO}$ terms, including biological processes, cellular components and molecular functions, and five KEGG signaling pathways were enriched for the 9 mRNAs, as indicated in Table SIV. GO terms and KEGG pathway analysis demonstrated that the 9 mRNAs associated gene sets regulated 'transmembrane receptor protein serine/threonine kinase signaling pathway', 'BMP signaling pathway', 'cation channel complex' and 'ion channel complex', 'RNA polymerase II core promoter sequence-specific DNA binding', 'RNA polymerase II transcription coactivator activity', 'nicotine addiction and amphetamine addiction'.

Expression of the genes analyzed in PCa and normal prostate cell lines. To verify whether the genes identified in the PCasig 
Table II. Cox regression analysis for PCasig and clinicopathological variables in the GS=7 subgroup from The Cancer Genome Atlas dataset.

$$
\text { Univariate Cox }
$$

\begin{tabular}{|c|c|c|c|c|}
\hline \multirow[b]{2}{*}{ Variable and intercept } & \multicolumn{2}{|c|}{ Univariate Cox } & \multicolumn{2}{|c|}{ Multivariate Cox } \\
\hline & HR $(95 \% \mathrm{CI})$ & P-value & HR $(95 \% \mathrm{CI})$ & P-value \\
\hline \multicolumn{5}{|l|}{ Age, years } \\
\hline$\leq 65$ & 1.00 & & NA & \\
\hline$>65$ & $2.48(0.88-7.00)$ & 0.08500 & NA & \\
\hline \multicolumn{5}{|l|}{ PT } \\
\hline $\mathrm{T} 2$ & 1.00 & & 1.00 & \\
\hline $\mathrm{T} 3 \mathrm{a}$ & $5.20(1.45-18.80)$ & 0.01100 & $3.87(1.04-14.30)$ & 0.04300 \\
\hline $\mathrm{T} 3 \mathrm{~b}+\mathrm{T} 4$ & $4.40(1.00-19.00)$ & 0.05100 & $2.24(0.41-12.10)$ & 0.34000 \\
\hline \multicolumn{5}{|l|}{$\mathrm{PN}$} \\
\hline No & 1.00 & & 1.00 & \\
\hline $\mathrm{N} 1$ & $6.22(2.19-17.60)$ & $<0.00010$ & $4.70(1.33-16.80)$ & 0.01600 \\
\hline NX & $8.20(0.070-4.19)$ & 0.56000 & $0.10(0.11-6.43)$ & 0.85000 \\
\hline \multicolumn{5}{|l|}{ Clinical M } \\
\hline M0 & 1.00 & & NA & \\
\hline M1 & $3.90 \times 10^{-8}(0.00-2.43)$ & 0.99000 & NA & \\
\hline \multicolumn{5}{|l|}{ Residual tumor } \\
\hline R0 & 1.00 & & & \\
\hline $\mathrm{R} 1$ or $\mathrm{R} 2$ & $0.50(0.12-2.00)$ & 0.36200 & NA & \\
\hline $\mathrm{RX}$ & $3.30 \times 10^{-8}(0.00-1.97)$ & 0.99800 & NA & \\
\hline \multicolumn{5}{|l|}{ Risk score } \\
\hline Low & 1.00 & & 1.00 & \\
\hline High & $13.00(3.13-12.91)$ & $<0.00010$ & $9.29(2.08-41.30)$ & 0.00340 \\
\hline
\end{tabular}

$\mathrm{N}=201$. NA indicates that the variable was not analyzed using multivariate Cox regression. HR, hazard ratio; GS, Gleason score; PT, pathological tumor grade; PN, pathological lymph node grade; pNX, patients did not undergo lymph node dissection; M, metastasis; $\mathrm{R}$, residual tumor stage; $\mathrm{RX}$, residual tumor not examined.

might be involved in PCa oncogenesis, the endogenous mRNA expression of all 9 genes was measured in five tumor-derived PCa cell lines (PC-3, DU145, 22RV1, LNCaP and C4-2) and a normal prostate cell line (RWPE-1) by quantitative PCR analysis. The results demonstrated that these genes exhibited relatively different expression in PCa cells compared with in normal prostate cell lines (Fig. 9).

\section{Discussion}

BCR prognostic tools are essential for the improvement of current treatment options for PCa and to reduce PCa-associated mortality for patients experiencing BCR following RP (5). Although previous studies have identified numerous potential biomarkers associated with prognosis and PCa progression, few of these markers have been applied in a clinical setting (20).

In the present study, the PCasig was developed using Cox regression and LASSO Cox analyses for the individualized prediction of BCR in patients with $\mathrm{GS} \geq 7$ from a TCGA dataset and validated in an Oncomine cohort. The PCasig consisted of 10 genes, including SEMG2, SOX11, AFP, KCNJ16, TFAP2B, SYCE1, KCNU1, GUCY1B2, GRIA4 and NXPH1. The signature may be used to classify patients with $\mathrm{PCa}$ into groups with low- and high-risk of BCR, which had significant differences in 3- and 5-year BCR-free survival time, with AUCs of 0.826 and 0.871 , respectively. These results suggested that the PCasig could serve as a predictor of BCR-free survival. Additionally, a clinical nomogram was constructed for comparison with the PCasig in the TCGA cohort, revealing improved performance of the clinical nomogram after incorporation of the PCasig (the PCasig vs. clinical nomogram; AUC, 0.835 vs. 0.764; C-index: 0.777 vs. 0.713 ). To assess the independence of the PCasig in predicting BCR-free survival, univariate and multivariate Cox regression analyses were performed and the risk scores of patients based on the PCasig maintained a good association with BCR-free survival. The PCasig was combined with clinicopathological data to construct a nomogram to predict BCR after RP. Incorporating the PCasig and clinical factors into the nomogram significantly improved the performance of the clinical nomogram in predicting BCR after RP, facilitating the construction of an individualized treatment for BCR after RP. The NRI (NRI, 0.75; P<0.001, event NRI, 0.66; non-event NRI, 0.09) indicated that performance was significantly improved following the integration of the PCasig into the clinical nomogram. The subgroup analysis further indicated a good prognostic value of the PCasig, regardless of GS. In 
Table III. Cox regression analysis for PCasig and clinicopathological variables in the GS $\geq 8$ subgroup from The Cancer Genome Atlas dataset.

\begin{tabular}{|c|c|c|c|c|}
\hline \multirow[b]{2}{*}{ Variable and intercept } & \multicolumn{2}{|c|}{ Univariate Cox } & \multicolumn{2}{|c|}{ Multivariate Cox } \\
\hline & HR $(95 \% \mathrm{CI})$ & P-value & HR $(95 \% \mathrm{CI})$ & P-value \\
\hline \multicolumn{5}{|l|}{ Age, years } \\
\hline$\leq 65$ & 1.00 & & NA & \\
\hline$>65$ & $0.90(0.51-1.56)$ & 0.71400 & NA & \\
\hline \multicolumn{5}{|l|}{ PT } \\
\hline $\mathrm{T} 2$ & 1.00 & & 1.00 & \\
\hline $\mathrm{T} 3 \mathrm{a}$ & $2.23(0.64-7.70)$ & 0.01100 & $2.40(0.69-8.40)$ & 0.16000 \\
\hline $\mathrm{T} 3 \mathrm{~b}+\mathrm{T} 4$ & $3.88(1.19-12.60)$ & 0.02400 & $4.02(1.20-13.10)$ & 0.02100 \\
\hline \multicolumn{5}{|l|}{$\mathrm{PN}$} \\
\hline No & 1.00 & & NA & \\
\hline N1 & $0.98(0.54-1.77)$ & 0.95000 & NA & \\
\hline NX & $0.29(0.04-2.18)$ & 0.23000 & NA & \\
\hline \multicolumn{5}{|l|}{ Clinical M } \\
\hline M0 & 1.00 & & NA & \\
\hline M1 & $0.43(0.050-3.15)$ & 0.41000 & NA & \\
\hline \multicolumn{5}{|l|}{ Residual tumor } \\
\hline R0 & 1.00 & & NA & \\
\hline $\mathrm{R} 1$ or $\mathrm{R} 2$ & $1.59(0.92-2.77)$ & 0.09000 & NA & \\
\hline $\mathrm{RX}$ & $0.24(0.03-1.80)$ & 0.17000 & NA & \\
\hline \multicolumn{5}{|l|}{ Risk score } \\
\hline Low & 1.00 & & 1.00 & \\
\hline High & $3.00(1.57-5.73)$ & 0.00082 & $3.01(1.58-5.75)$ & 0.00080 \\
\hline
\end{tabular}

$\mathrm{N}=176$. NA indicates that the variable was not analyzed using multivariate Cox regression. HR, hazard ratio; GS, Gleason Score; PT, pathological tumor grade; PN, pathological lymph node grade; pNX, patients did not undergo lymph node dissection; M, metastasis; R, residual tumor stage; $\mathrm{RX}$, residual tumor not examined.

addition, the endogenous mRNA expression of genes in the PCasig were measured in PCa cell lines and normal prostate cells, providing a basis for further functional studies on the role of these genes in PCa. Overall, the present findings highlighted the added value of the PCasig for the prediction of $\mathrm{BCR}$ in patients with $\mathrm{PCa}$.

Previous studies have investigated the functional role of the 10 genes in the PCasig in cancer progression. For example, SEMG2 has been previously identified as a biomarker for lung cancer (21). SOX11 acts as a transcriptional regulator after forming a protein complex with other proteins. SOX11 overexpression can suppress proliferation, invasion and migration of PCa cells in vitro (22). AFP is a major plasma protein produced by the yolk sac and the liver during fetal life. The aberrant expression in adults is often associated with hepatoma or teratoma (23). It has been previously reported that AFP is negatively regulated by AT-binding transcription factor 1 (ATBF1) (24). Sun et al (25), reported that loss of ATBF1 is one mechanism that defines the progression in PCa. KCNJ16 is a potassium channel gene, which functions in fluid and $\mathrm{pH}$ balance regulation. Downregulation of KCNJ16 may lead to an imbalance in ion concentration between the extracellular and intracellular compartments, and influence tumor progression through multiple paths, including cell adhesion or migration, angiogenesis and apoptosis (26). TFAP2B transcription factor is a member of the AP-2 family, which serves an important role in cell apoptosis and autophagy (27). TFAP2B is also detectable in endometrial cancer, and patients with low TFAP2B expression have a worse prognosis compared with patients with high TFAP2B expression (28). SYCE1 consists of two lateral elements and a central region formed by transverse elements and a central element. In addition, SYCE1 may interact with chromosome 14 open reading frame 39 serving a role in the early stages of meiosis and the cell cycle (29). Furthermore, it has been suggested that SYCE1 may be a promising immunotherapy approach for lung adenocarcinoma (30). The potassium voltage-gated ion channel KCNU1 is regulated by calcium ion levels, which is critical for human fertility (31). KCNU1 may be activated by both intracellular $\mathrm{pH}$ and membrane voltage that mediates the export of $\mathrm{K}^{+}(32)$. GUCY1B2 is considered a pseudogene with a frameshift mutation on the Genbank website (https://cipotato.org/genebankcip/) and its role in PCa is not yet fully understood (33). Excitatory neurotransmitter receptor GRIA4 has been identified as a biomarker for colorectal cancer, which may vary in signal transduction properties $(34,35)$. NXPH1 functions as a 


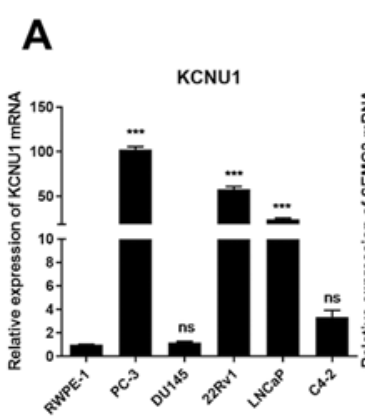

B

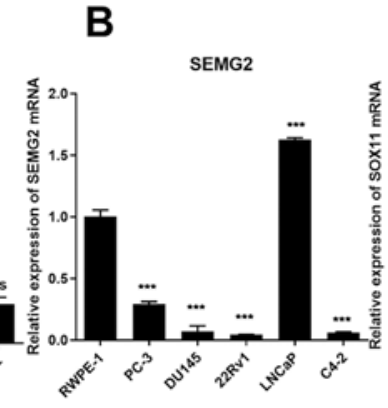

C

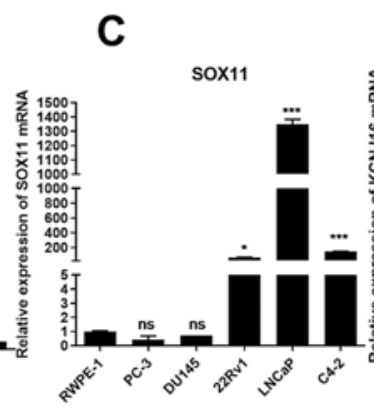

D

KCNJ16

E
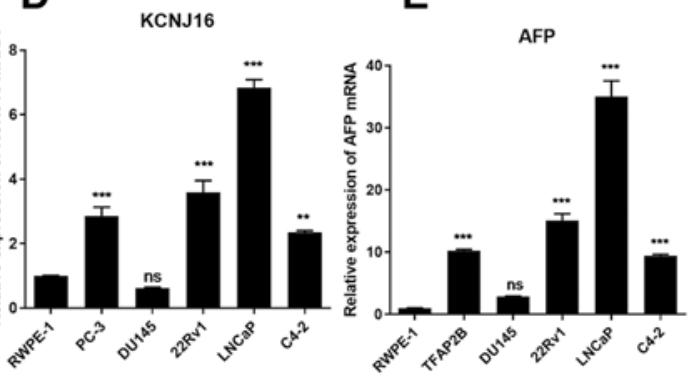

F

G

H

I

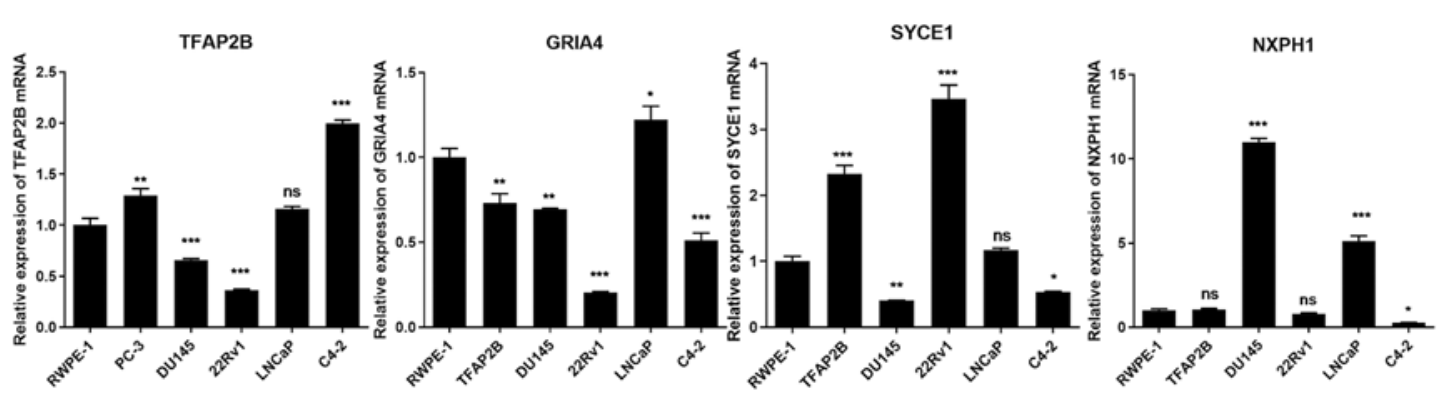

Figure 9. Endogenous expression of the 9 mRNAs. Expression of 9 mRNAs was measured using reverse transcription-quantitative PCR in five prostate cancer (PC-3, DU145, 22Rv1, LNCaP and C4-2) and one normal prostate (RWPE-1) cell lines. GAPDH was used as an internal reference. "P<0.05, ${ }^{* *} \mathrm{P}<0.01,{ }^{* * *} \mathrm{P}<0.001$ vs. RWPE-1. (A) KCNU1, (B) SEMG2, (C) SOX11 (D) KCNJ16, (E) AFP, (F) TFAP2B, (G) GRIA4, (H) SYCE1, (I) NXPH1. ns, not significant; KCNU1, potassium calcium-activated channel subfamily U member 1; SEMG2, semenogelin 2; SOX11, SRY-box transcription factor 11; KCNJ16, potassium inwardly rectifying channel subfamily J member 16; AFP, $\alpha$-fetoprotein; TFAP2B, transcription factor AP-2 $\beta$; GRIA4, glutamate ionotropic receptor AMPA type subunit 4; SYCE1, synaptonemal complex central element protein 1; NXPH1, neurexophilin 1.

secreted protein that promotes cell adhesion (36). High expression of NXPH1 is associated with poor prognosis in patients with breast cancer (37). Therefore, since these genes appear to serve several biological functions in multiple human malignancies, understanding the underlying role of each of these 10 genes in PCa would provide novel insights into the functional relevance of the PCasig.

However, there are some limitations in the present study. Firstly, since TCGA and Oncomine lack data regarding pre-surgical serum PSA, an association between PSA and BCR could not be determined. Secondly, future experimental studies should be conducted to examine the functional role of the genes identified in the present study and validate their expression at the protein level.

In conclusion, the present study identified a gene cluster that may act as an independent prognostic factor, or signature, with greater prognostic value for all patients regardless of GS, which can significantly improve the performance of clinical nomograms in predicting BCR of patients with $\mathrm{PCa}$ and $\mathrm{GS} \geq 7$.

\section{Acknowledgements}

Not applicable.

\section{Funding}

The present study was supported by The National Natural Science Foundation of China (grant no. 81600542), The Guangdong Basic and Applied Basic Research Foundation (grant no. 2019A1515110033), The Distinguished Young Talents in Higher Education Foundation of Guangdong
Province (grant no. 2019KQNCX115), China Postdoctoral Science Foundation (grant no. 2019M662865) and achievement cultivation and clinical transformation application cultivation projects of the First Affiliated Hospital of Guangzhou Medical University (grant no. ZH201908). Additional funding was provided by the Guangzhou Science Technology and Innovation Commission (grant nos. 201604020001 and No.201704020193), and The Project of Health and Family Planning Commission of Guangzhou Municipality (grant no. 20181A010051).

\section{Availability of data and materials}

The datasets generated and/or analyzed during the current study are available in the TCGA repository (https://portal.gdc. cancer.gov) and Oncomine website (https://www.oncomine. org/resource/main.html).

\section{Authors' contributions}

YL and DG conceived of the study and participated in its design and coordination. XW, DL, ML, CC, ZZ and ME contributed to analysis and interpretation of data and were involved in drafting the manuscript. All authors read and approved the final manuscript.

\section{Ethics approval and consent to participate}

Not applicable.

\section{Patient consent for publication}

Not applicable. 


\section{Competing interests}

The authors declare that they have no competing interests.

\section{References}

1. Siegel RL, Miller KD and Jemal A: Cancer statistics, 2020. CA Cancer J Clin 70: 7-30, 2020

2. Chen W, Zheng R, Baade PD, Zhang S, Zeng H, Bray F, Jemal A, Yu XQ and He J: Cancer statistics in China, 2015. CA Cancer J Clin 66: 115-132, 2016.

3. Lalonde E, Ishkanian AS, Sykes J, Fraser M, Ross-Adams H, Erho N, Dunning MJ, Halim S, Lamb AD, Moon NC, et al: Tumour genomic and microenvironmental heterogeneity for integrated prediction of 5-year biochemical recurrence of prostate cancer: A retrospective cohort study. Lancet Oncol 15: 1521-1532, 2014.

4. Van den Broeck T, van den Bergh RCN, Arfi N, Gross T, Moris L, Briers E, Cumberbatch M, De Santis M, Tilki D, Fanti S, et al Prognostic value of biochemical recurrence following treatment with curative intent for prostate cancer: A systematic review. Eur Urol 75: 967-987, 2019.

5. Brockman JA, Alanee S, Vickers AJ, Scardino PT, Wood DP, Kibel AS, Lin DW, Bianco FJ Jr, Rabah DM, Klein EA, et al Nomogram predicting prostate cancer-specific mortality for men with biochemical recurrence after radical prostatectomy. Eur Urol 67: 1160-1167, 2015.

6. Fraser M, Berlin A, Bristow RG and van der Kwast T: Genomic, pathological, and clinical heterogeneity as drivers of personalized medicine in prostate cancer. Urol Onco 33: 85-94, 2015.

7. Burke HB: Predicting clinical outcomes using molecular biomarkers. Biomark Cancer 8: 89-99, 2016.

8. Amaro A, Esposito AI, Gallina A, Nees M, Angelini G, Albini A and Pfeffer U: Validation of proposed prostate cancer biomarkers with gene expression data: A long road to travel. Cancer Metastasis Rev 33: 657-671, 2014

9. Gleason DF and Mellinger GT: Prediction of prognosis for prostatic adenocarcinoma by combined histological grading and clinical staging. J Urol 111: 58-64, 1974.

10. Ross HM, Kryvenko ON, Cowan JE, Simko JP, Wheeler TM and Epstein JI: Do adenocarcinomas of the prostate with Gleason score $(\mathrm{GS})</=6$ have the potential to metastasize to lymph nodes? Am J Surg Pathol 36: 1346-1352, 2012.

11. Epstein JI, Zelefsky MJ, Sjoberg DD, Nelson JB, Egevad L, Magi-Galluzzi C, Vickers AJ, Parwani AV, Reuter VE, Fine SW, et al: A contemporary prostate cancer grading system: A validated alternative to the gleason score. Eur Urol 69: $428-435,2016$

12. Esserman LJ, Thompson IM, Reid B, Nelson P, Ransohoff DF, Welch HG, Hwang S, Berry DA, Kinzler KW, Black WC, et al: Addressing overdiagnosis and overtreatment in cancer: A prescription for change. Lancet Oncol 15: e234-e242, 2014.

13. Yin Y, Zhang Q, Zhang H, He Y and Huang J: Molecular signature to risk-stratify prostate cancer of intermediate risk. Clin Cancer Res 23: 6-8, 2017.

14. Beauval JB, Roumiguié M, Filleron T, Benoit T, de la Taille A Malavaud B, Salomon L, Soulié M and Ploussard G: Biochemical recurrence-free survival and pathological outcomes after radical prostatectomy for high-risk prostate cancer. BMC Urol 16: 26, 2016.

15. Gearman DJ, Morlacco A, Cheville JC, Rangel LJ and Karnes RJ: Comparison of pathological and oncologic outcomes of favorable risk gleason score $3+4$ and low risk gleason score 6 prostate cancer: Considerations for active surveillance. J Urol 199: 1188-1195, 2018.

16. Mohler JL, Antonarakis ES, Armstrong AJ, D'Amico AV, Davis BJ, Dorff T, Eastham JA, Enke CA, Farrington TA, Higano CS, et al: Prostate cancer, version 2.2019, NCCN clinical practice guidelines in oncology. J Natl Compr Canc Netw 17: 479-505, 2019.

17. Kaneko S, Hirakawa A and Hamada C: Enhancing the lasso approach for developing a survival prediction model based on gene expression data. Comput Math Methods Med 2015: 259474, 2015.

18. Pencina MJ, D'Agostino RB Sr and Steyerberg EW: Extensions of net reclassification improvement calculations to measure usefulness of new biomarkers. Stat Med 30: 11-21, 2011

19. Livak KJ and Schmittgen TD: Analysis of relative gene expression data using real-time quantitative PCR and the 2(-Delta Delta C(T)) method. Methods 25: 402-408, 2001.
20. Abou-Ouf H, Alshalalfa M, Takhar M, Erho N, Donnelly B, Davicioni E, Karnes RJ and Bismar TA: Validation of a 10-gene molecular signature for predicting biochemical recurrence and clinical metastasis in localized prostate cancer. J Cancer Res Clin Oncol 144: 883-891, 2018.

21. Luo WM, Wang ZY and Zhang X: Identification of four differentially methylated genes as prognostic signatures for stage I lung adenocarcinoma. Cancer Cell Int 18: 60, 2018.

22. Yao Z, Sun B, Hong Q, Yan J, Mu D, Li J, Sheng H and Guo H: The role of tumor suppressor gene SOX11 in prostate cancer. Tumour Biol 36: 6133-6138, 2015.

23. Hirschfield H, Bian CB, Higashi T, Nakagawa S, Zeleke TZ, Nair VD, Fuchs BC and Hoshida Y: In vitro modeling of hepatocellular carcinoma molecular subtypes for anti-cancer drug assessment. Exp Mol Med 50: e419, 2018

24. Ninomiya T, Mihara K, Fushimi K, Hayashi Y, Hashimoto-Tamaoki T and Tamaoki T: Regulation of the alpha-fetoprotein gene by the isoforms of ATBF1 transcription factor in human hepatoma. Hepatology 35: 82-87, 2002.

25. Sun X, Frierson HF, Chen C, Li C, Ran Q, Otto KB, Cantarel BL, Vessella RL, Gao AC, Petros J, et al: Frequent somatic mutations of the transcription factor ATBF1 in human prostate cancer. Nat Genet 37: 407-412, 2005.

26. Pardo LA and Stühmer W: The roles of $\mathrm{K}(+)$ channels in cancer. Nat Rev Cancer 14: 39-48, 2014

27. Ren KW, Li YH, Wu G, Ren JZ, Lu HB, Li ZM and Han XW: Quercetin nanoparticles display antitumor activity via proliferation inhibition and apoptosis induction in liver cancer cells. Int J Oncol 50: 1299-1311, 2017.

28. Wu H and Zhang J: Decreased expression of TFAP2B in endometrial cancer predicts poor prognosis: A study based on TCGA data. Gynecol Oncol 149: 592-597, 2018.

29. Gómez HL, Felipe-Medina N, Sánchez-Martin M, Davies OR, Ramos I, García-Tuñón I, de Rooij DG, Dereli I, Tóth A, Benavente R, et al: C14ORF39/SIX6OS1 is a constituent of the synaptonemal complex and is essential for mouse fertility. Nat Commun 7: 13298, 2016.

30. Taguchi A, Taylor AD, Rodriguez J, Celiktas M, Liu H, Ma X, Zhang Z, Wong CH, Chin H, Girard L, et al: A search for novel cancer/testis antigens in lung cancer identifies VCX/Y genes, expanding the repertoire of potential immunotherapeutic targets. Cancer Res 74: 4694-4705, 2014.

31. Geng Y, Ferreira JJ, Dzikunu V, Butler A, Lybaert P, Yuan P, Magleby KL, Salkoff L and Santi CM: A genetic variant of the sperm-specific SLO3 $\mathrm{K}^{+}$channel has altered $\mathrm{pH}$ and $\mathrm{Ca}^{2+}$ sensitivities. J Biol Chem 292: 8978-8987, 2017.

32. Shukla KK, Mahdi AA and Rajender S: Ion channels in sperm physiology and male fertility and infertility. J Androl 33: 777-788, 2012.

33. Bossini-Castillo L, de Kovel C, Kallberg H, van 't Slot R, Italiaander A, Coenen M, Tak PP, Posthumus MD, Wijmenga C, Huizinga T, et al: A genome-wide association study of rheumatoid arthritis without antibodies against citrullinated peptides. Ann Rheum Dis 74: e15, 2015.

34. Hauptman N, Jevšinek Skok D, Spasovska E, Boštjančič E and Glavač D: Genes CEP55, FOXD3, FOXF2, GNAO1, GRIA4, and KCNA5 as potential diagnostic biomarkers in colorectal cancer. BMC Med Genomics 12: 54, 2019.

35. Barault L, Amatu A, Siravegna G, Ponzetti A, Moran S, Cassingena A, Mussolin B, Falcomatà C, Binder AM, Cristiano $\mathrm{C}$, et al: Discovery of methylated circulating DNA biomarkers for comprehensive non-invasive monitoring of treatment response in metastatic colorectal cancer. Gut 67: 1995-2005, 2018.

36. Born G, Breuer D, Wang S, Rohlmann A, Coulon P, Vakili P, ReissnerC,Kiefer F,Heine M,Pape HCand Missler M: Modulation of synaptic function through the alpha-neurexin-specific ligand neurexophilin-1. Proc Natl Acad Sci USA 111: E1274-E1283, 2014.

37. Faryna M, Konermann C, Aulmann S, Bermejo JL, Brugger M, Diederichs S, Rom J, Weichenhan D, Claus R, Rehli M, et al: Genome-wide methylation screen in low-grade breast cancer identifies novel epigenetically altered genes as potential biomarkers for tumor diagnosis. FASEB J 26: 4937-4950, 2012.

This work is licensed under a Creative Commons Attribution-NonCommercial-NoDerivatives 4.0 International (CC BY-NC-ND 4.0) License. 